\title{
Recovery from chronic fatigue syndrome after treatments given in the PACE trial
}

\author{
P. D. White ${ }^{1 *}$, K. Goldsmith ${ }^{2}$, A. L. Johnson ${ }^{3,4}$, T. Chalder $^{5}$ and M. Sharpe ${ }^{6}$; PACE Trial Management \\ Group $\dagger$ \\ ${ }^{1}$ Wolfson Institute of Preventive Medicine, Barts and the London School of Medicine and Dentistry, Queen Mary University of London, UK \\ ${ }^{2}$ Biostatistics Department, Institute of Psychiatry, King's College London, UK \\ ${ }^{3}$ MRC Biostatistics Unit, Institute of Public Health, University of Cambridge, UK \\ ${ }^{4}$ MRC Clinical Trials Unit, London, UK \\ ${ }^{5}$ Academic Department of Psychological Medicine, King's College London, UK \\ ${ }^{6}$ Department of Psychiatry, University of Oxford, UK
}

Background. A multi-centre, four-arm trial (the PACE trial) found that rehabilitative cognitive behaviour therapy (CBT) and graded exercise therapy (GET) were more effective treatments for chronic fatigue syndrome (CFS) than specialist medical care (SMC) alone, when each was added to SMC, and more effective than adaptive pacing therapy (APT) when added to SMC. In this study we compared how many participants recovered after each treatment.

\begin{abstract}
Method. We defined recovery operationally using multiple criteria, and compared the proportions of participants meeting each individual criterion along with two composite criteria, defined as (a) recovery in the context of the trial and $(b)$ clinical recovery from the current episode of the illness, however defined, 52 weeks after randomization. We used logistic regression modelling to compare treatments.
\end{abstract}

Results. The percentages (number/total) meeting trial criteria for recovery were $22 \%(32 / 143)$ after CBT, $22 \%$ $(32 / 143)$ after GET, $8 \%(12 / 149)$ after APT and 7\% (11/150) after SMC. Similar proportions met criteria for clinical recovery. The odds ratio (OR) for trial recovery after CBT was 3.36 [95\% confidence interval (CI) 1.64-6.88] and for GET 3.38 (95\% CI 1.65-6.93), when compared to APT, and after CBT 3.69 (95\% CI 1.77-7.69) and GET 3.71 (95\% CI 1.78-7.74), when compared to SMC ( $p$ values $\leqslant 0.001$ for all comparisons). There was no significant difference between APT and SMC. Similar proportions recovered in trial subgroups meeting different definitions of the illness.

Conclusions. This study confirms that recovery from CFS is possible, and that CBT and GET are the therapies most likely to lead to recovery.

Received 16 August 2012; Revised 14 December 2012; Accepted 17 December 2012; First published online 31 January 2013

Key words: Chronic fatigue syndrome, cognitive behaviour therapy, graded exercise therapy, randomized control trial, recovery.

\section{Introduction}

Chronic fatigue syndrome (CFS) is a disabling disorder of unknown cause, with a prevalence of between $0.4 \%$ and $2.5 \%$ in the UK population (Prins et al. 2006). Myalgic encephalomyelitis (ME) is thought by some researchers to be the same disorder and by others as different with separate diagnostic criteria (Prins et al. 2006; NICE, 2007). Common symptoms of CFS include fatigue, painful muscles and joints, poor concentration

* Address for correspondence: Professor P. D. White, Department of Psychological Medicine, St Bartholomew's Hospital, London EC1A 7BE, UK.

(Email: p.d.white@qmul.ac.uk)

$\dagger$ Members of the PACE Trial Management Group are given in the Appendix. and sleep disturbance; these symptoms do not remit with rest and are made worse by activity.

Established treatments for CFS include the rehabilitative therapies of cognitive behaviour therapy (CBT) and graded exercise therapy (GET) (NICE, 2007). Several meta-analyses of these therapies indicate moderate benefit from these treatments (Edmonds et al. 2004; Malouff et al. 2008; Price et al. 2008; Castell et al. 2011). The recently published PACE trial found that CBT and GET were more effective in reducing both fatigue and physical disability than adaptive pacing therapy (APT), when each was added to specialist medical care (SMC), and more effective than SMC alone (White et al. 2011).

Although the PACE trial found that many patients improved with CBT and GET, the question of how many patients recovered remains unanswered. We 
know that recovery from CFS without treatment is reported to be uncommon; a systematic review found that a median (range) of only $7 \%(0-48 \%)$ recovered over time (Cairns \& Hotopf, 2005). We also know from a previous study that $24 \%$ of 25 patients rated themselves as 'recovered' 5 years after CBT (Deale et al. 2001) and that when applying more detailed, operationalized criteria (no longer fatigued, able to resume activities, and a perception of health and fatigue similar to that of a healthy person), $23 \%$ of 96 patients were rated as 'recovered' immediately after a course of CBT (Knoop et al. 2007). However, there have been no published reports comparing the proportions recovered after CBT with those achieved after other treatments.

Before we can determine the proportions recovered we need an operational definition of recovery itself. An ideal definition remains uncertain, as is the case for other conditions, such as low back pain (Kamper et al. 2011). Measurement of recovery could involve many domains. Within the trial context these could include: no longer meeting trial eligibility criteria, not having significant symptoms, not being disabled by the illness, and regarding one's health as having improved considerably. Within a clinical context, the additional criteria of not meeting alternative definitions of CFS and ME could also be applied (Sharpe et al. 1991; Tyrrell, 1994; Reeves et al. 2003).

Creating criteria for recovery from domains that are measured on a continuum requires the setting of operational thresholds based on population studies or trial eligibility criteria (Powell et al. 2004; Knoop et al. 2007; Malouff et al. 2008). In this context it is important to note that recovery does not mean being free of all symptoms; population studies show that the average person in the UK reports a mean of four symptoms in any 2-week period (McAteer et al. 2011). The three most common symptoms reported were fatigue, headache and joint pain; symptoms consistent with CFS (McAteer et al. 2011). Recovery may be taken to imply that the patient has made a transition from ill health to remission and also is at little risk of recurrence (Nisenbaum et al. 2003). Although we can measure remission, we cannot be certain of the risk of recurrence without long-term follow-up; we therefore use the term 'recovery' in this paper to mean recovery from the current episode of the illness.

The aims of this study were to: $(a)$ define operationalized criteria for recovery on relevant domains, (b) calculate the proportions of trial participants meeting each of these individual criteria in each treatment arm, (c) calculate the proportion of trial participants meeting all the recovery criteria to provide a comprehensive and conservative definition of recovery in each treatment arm, $(d)$ compare the proportions meeting both trial and clinical recovery criteria between the treatment arms, and (e) compare these proportions within each of the two subgroups of participants in the trial, which met the international definition of CFS and the London definition of ME (Tyrrell, 1994). As CBT and GET were the most effective treatments in the trial, we hypothesized that they would also be associated with greater proportions of recovered individuals at the 52-week primary end-point than either APT or SMC alone.

\section{Method}

The PACE trial recruited 641 participants from six secondary care CFS clinics in England and Scotland, allocated randomly to one of four treatment groups, with a final follow-up 52 weeks after randomization (White et al. 2007, 2011). All participants met the Oxford criteria for CFS (Sharpe et al. 1991). The four trial treatment arms were: SMC alone delivered by specialist CFS doctors, SMC plus APT delivered by occupational therapists, SMC plus CBT delivered by clinical psychologists, and SMC plus GET delivered by physiotherapists. Specialist doctors gave an explanation of why participants were ill and general advice about managing the illness. They also prescribed medicines to help with symptoms such as insomnia and pain, or advised general practitioners (GPs) on which medicine was appropriate. If a participant was randomized to this treatment alone, they were also encouraged to use self-help management that made most sense to them. APT involved carefully matching activity levels to the amount of energy available. Therapists worked with participants in this group to help monitor activity and symptoms, aiming to improve quality of life and create the best conditions for natural remission. CBT involved examining how thoughts, behaviour and symptoms interact with each other. Between therapy sessions, participants in this group were encouraged to try out new ways of coping with their illness. GET involved gradually increasing physical activity to improve fitness and get the body used to activity again. Therapists helped participants in this group to work out a basic activity routine and slowly build up the amount of exercise (White et al. 2007, 2011).

\section{Domains, measures and criteria for defining recovery}

We chose domains for defining recovery on the basis of the previous literature and the measures available from the trial. The thresholds defining our criteria for recovery on each domain were based either on population normal ranges, case definitions or trial entry criteria. We changed three of the thresholds for 
measuring recovery from our original protocol (White et al. 2007) before the analysis, as explained below.

\section{Fatigue: the Chalder Fatigue Questionnaire (CFQ)}

The 11-item CFQ measured the severity of symptomatic fatigue, rated by the participant, and was one of the primary outcomes of the trial (Chalder et al. 1993). The respondent chose from one of four answers ('less than usual', 'no more than usual', 'more than usual' and 'much more than usual') to each item, scores being $0,1,2$ or 3 , with a maximum score of 33 indicating severe fatigue. We changed our original protocol's threshold score for being within a normal range from a binary score of $\leqslant 3$ out of 11 (White et al. 2007), which represented a screening threshold for abnormal fatigue from a small primary care study (Chalder et al. 1993), following the publication of a much larger study of fatigue in adults in a representative population sample of patients registered with a GP from South East England (Cella \& Chalder, 2010). This showed a population mean (S.D.) Likert score of 14.2 (4.6) out of a maximum score of 33 . We therefore considered a score of 18 (highest integral score below the mean plus 1 s.D.) or less as within the normal range for fatigue.

Physical function: the 36-Item Short-Form Health Survey (SF-36) physical function subscale

The SF-36 physical function subscale, rated by the participants, was the other primary outcome from the trial (McHorney et al. 1993). The scale asks about 10 aspects of physical function, such as the ability to walk $100 \mathrm{~m}$, with three possible answers: not limited, limited a little, and limited a lot. This provides a derived score that ranges from worst (0) to best possible function (100). We changed our original protocol's threshold score for being within a normal range on this measure from a score of $\geqslant 85$ to a lower score as that threshold would mean that approximately half the general working age population would fall outside the normal range. The mean (s.D.) scores for a demographically representative English adult population were 86.3 (22.5) for males and 81.8 (25.7) for females (Bowling et al. 1999). We derived a mean (s.D.) score of 84 (24) for the whole sample, giving a normal range of 60 or above for physical function.

\section{CFS case definition: Oxford criteria}

This was the definition of CFS used to define eligibility for participation in the trial. Research assessors judged whether participants still met Oxford criteria for CFS at 52 weeks; specifically they determined if: (1) fatigue was the main symptom, (2) it was of definite onset and not lifelong, (3) fatigue was severe, disabling and affected physical and mental function, and (4) fatigue had persisted for 6 months or more and was present $50 \%$ of the time (Sharpe et al. 1991). To satisfy the third criterion for severity of fatigue and disability, participants had to meet trial entry thresholds for fatigue (a binary score of $\geqslant 6$ out of 11 on the CFQ) and abnormal levels of physical function (a score of $\leqslant 65$ out of 100 on the SF-36 physical function subscale) (White et al. 2007).

\section{CFS case definition: the International (Centers for Disease Control and Prevention, CDC) criteria}

The research assessor used participant ratings to judge whether participants met the International (CDC) criteria for CFS at 52 weeks (Reeves et al. 2003), which included: (1) severe chronic fatigue for at least 6 months with other known medical conditions (whose manifestation includes fatigue) excluded by clinical diagnosis; and (2) concurrently have four or more of the following symptoms: post-exertional malaise, impaired memory or concentration, unrefreshing sleep, muscle pain, multi-joint pain without redness or swelling, tender cervical or axillary lymph nodes, sore throat, headache. For the purposes of this study, the four or more symptoms needed to be present within the previous week of the assessment date, rather than the previous 6 months (Reeves et al. 2003). To meet the first criterion for severity, participants had to have abnormal levels of fatigue, which we took to be the trial entry eligibility criteria for the CFQ, and abnormal levels of physical function (as above) (White et al. 2007).

\section{ME case definition: the London criteria}

Research assessors judged whether participants met the London criteria for ME at 52 weeks (Tyrrell, 1994). Specifically, these criteria included: (1) exerciseinduced fatigue precipitated by trivially small exertion, (2) impairment of short-term memory and loss of powers of concentration, (3) fluctuations of symptoms usually precipitated by physical or mental exertion, (4) symptoms present for at least 6 months, and (5) no 'primary' depressive illness and no anxiety disorder present (which we interpreted as no co-morbid mood disorder of any kind). To standardize thresholds for severity with other case definitions, participants also had to meet trial entry eligibility criteria for the CFQ and abnormal levels of physical function (as above) (White et al. 2007).

\section{Overall change in health: the Clinical Global Impression (CGI) change score}

The self-rated CGI change score (range 1-7) provided a participant-rated global measure of overall health 
change, not just change in CFS (Guy, 1976). We considered scores of 1 ('very much better') or 2 ('much better') as evidence of the process of recovery, rather than our original protocol threshold of a score of 1 only, because we considered that participants rating their overall health as 'much better' represented the process of recovery. The CGI change scale was also rated by the SMC doctor at the 52-week review. These scores were used as imputed scores when the participant-rated CGI score was missing at 52 weeks $(n=22)$.

\section{Composite definitions of recovery}

We operationalized two composite definitions of recovery: (1) trial recovery from CFS, and (2) clinical recovery from the illness, however it was defined. To provide a definition of trial recovery, we calculated a hierarchical, cumulative definition that included the following domains mentioned earlier: normal range in fatigue, normal range in physical function, not meeting the Oxford case definition of CFS, and CGI scores of 1 or 2 ('very much' or 'much' better). To fulfil the criteria for clinical recovery from the illness, participants had to meet all the criteria for trialdefined recovery (described earlier), in addition to not meeting either the International (CDC) criteria for CFS or the London criteria for ME.

\section{Analysis}

We reported descriptive statistics (percentage and frequency) in each treatment arm for each individual domain of recovery. We then gave the results of a cumulative hierarchy of the proportions meeting domains of trial recovery in the order of: normal ranges for both the fatigue and physical function scores, not meeting the Oxford criteria for CFS, and the clinical global impression positive change scores (1 or 2$)$. The cumulative hierarchy of clinical recovery was then applied as the trial definition of recovery combined with not meeting criteria for either the International (CDC) definition of CFS or the London criteria for ME. We calculated the number needed to treat (NNT) for one extra participant to recover by dividing 100 by the proportion recovering after either CBT or GET minus the proportion recovering after $\mathrm{SMC}$, rounded up to the nearest whole number.

To examine recovery in participants who also met either the International (CDC) definition of CFS or the London definition of ME at entry to the trial, we applied the same cumulative hierarchy of criteria in these subgroups. We then used logistic regression to compare the odds of recovery between trial arms, using the originally hypothesized comparison groups:
APT v. SMC, CBT v. SMC, GET v. SMC, APT v. CBT and APT v. GET (White et al. 2011). Resulting odds ratios (ORs) and 95\% confidence intervals (CIs) were adjusted for the stratification variables of centre, meeting International (CDC) criteria at baseline, meeting the London definition of $\mathrm{ME}$ at baseline, and having a depressive illness at baseline. Interaction terms for trial arm by meeting either CFS or ME criteria at baseline were used to calculate the odds of recovery in (1) the subgroup meeting International (CDC) criteria at baseline and (2) the subgroup meeting the London definition of ME at baseline.

\section{Results}

We studied 640 participants (excluding one participant who withdrew consent after the study). The mean (s.D.) age was 38 (12) years, 77\% were female, and $93 \%$ were Caucasian. All participants met the Oxford criteria for CFS; $67 \%$ of participants also met the International (CDC) criteria for CFS and 51\% met the London criteria for ME (White et al. 2011). The median (interquartile range, IQR) duration of illness was 32 (16-68) months, $47 \%$ had a co-morbid psychiatric condition at randomization (33\% depressive disorder). By 52 weeks, only 33 (5\%) were missing primary outcome data, with no significant difference between treatment groups.

Table $1 a$ shows the individual criteria for recovery at 52 weeks. Whatever the domain, the largest proportions of participants recovering had received either CBT or GET. Overall, the largest proportions of participants meeting criteria for recovery were those who no longer met criteria for $\mathrm{ME}$, followed by the Internationally defined criteria for CFS, and then the Oxford-defined criteria for CFS.

Table $1 b$ shows the hierarchical, cumulative definitions for both trial and clinical recovery. As each additional criterion was added, the proportions meeting criteria for recovery generally were reduced. For all the criteria applied, the largest proportions of recovered participants were found in those who had received either CBT or GET. Some data were missing for $6 \%$ of those receiving APT and SMC and $11 \%$ for those in receipt of CBT and GET. The NNT for one extra participant to achieve trial recovery was 7 for both CBT and GET.

The proportions meeting criteria for clinical recovery from the illness were very similar to the proportions meeting the trial definition for recovery (Table $1 b$ ). Although it seemed that slightly smaller proportions had recovered from the illness as a whole, when the criterion 'not meeting the London criteria for $M E^{\prime}$ was applied, we found that the differences were due to missing data rather than to change in recovery 
Table 1. Participants, \% (n/total), meeting criteria for recovery

\begin{tabular}{|c|c|c|c|c|}
\hline Domains and measures & APT (159) & CBT (161) & GET (160) & SMC (160) \\
\hline \multicolumn{5}{|l|}{ (a) Individual criteria } \\
\hline \multicolumn{5}{|l|}{ Fatigue } \\
\hline Within CFQ normal range & $22(34 / 153)$ & $41(60 / 148)$ & $33(51 / 154)$ & $21(32 / 152)$ \\
\hline \multicolumn{5}{|l|}{ Physical function } \\
\hline Within SF-36-PF normal range & $35(53 / 153)$ & $52(77 / 148)$ & $53(81 / 154)$ & $41(62 / 152)$ \\
\hline \multicolumn{5}{|l|}{ Both fatigue and function } \\
\hline $\begin{array}{l}\text { Within both CFQ and SF-36-PF } \\
\text { normal ranges }\end{array}$ & $16(25 / 153)$ & $30(44 / 148)$ & $28(43 / 154)$ & $15(22 / 152)$ \\
\hline \multicolumn{5}{|l|}{ Case criteria } \\
\hline CDC criteria not met & $49(74 / 150)$ & $67(97 / 144)$ & $65(93 / 144)$ & $51(76 / 149)$ \\
\hline Oxford criteria not met & $43(64 / 149)$ & $54(77 / 143)$ & $56(81 / 144)$ & $41(62 / 150)$ \\
\hline London ME criteria not met & $68(100 / 147)$ & $76(107 / 140)$ & $77(106 / 138)$ & $66(97 / 148)$ \\
\hline \multicolumn{5}{|l|}{ Overall health change } \\
\hline \multirow[t]{2}{*}{ CGI 1 or 2} & $30(48 / 158)$ & $40(62 / 154)$ & $40(63 / 156)$ & $25(40 / 158)$ \\
\hline & $\mathrm{APT}$ & CBT & GET & SMC \\
\hline \multicolumn{5}{|c|}{ (b) Composite criteria for both trial and clinical recovery (combined hierarchically) } \\
\hline \multicolumn{5}{|l|}{ Cumulative criteria for trial recovery } \\
\hline $\begin{array}{l}\text { Both CFQ and SF-36-PF in normal } \\
\text { range }\end{array}$ & $16(25 / 153)$ & $30(44 / 148)$ & $28(43 / 154)$ & $15(22 / 152)$ \\
\hline And Oxford criteria not met & $15(23 / 149)$ & $28(40 / 143)$ & $28(41 / 144)$ & $14(21 / 150)$ \\
\hline And CGI 1 or $2(95 \%$ CI $)$ & $\begin{array}{l}8(12 / 149) \\
(4-14)\end{array}$ & $\begin{array}{c}22(32 / 143) \\
(16-30)\end{array}$ & $\begin{array}{c}22(32 / 143) \\
(16-30)\end{array}$ & $\begin{array}{l}7(11 / 150) \\
(4-13)\end{array}$ \\
\hline \multicolumn{5}{|l|}{ Additional criteria for clinical recovery } \\
\hline $\begin{array}{l}\text { And International (CDC) CFS criteria } \\
\text { not met }\end{array}$ & $8(12 / 149)$ & $22(32 / 143)$ & $22(32 / 143)$ & $7(11 / 149)$ \\
\hline $\begin{array}{l}\text { And London ME criteria not met } \\
(95 \% \mathrm{CI})\end{array}$ & $\begin{array}{l}8(12 / 147) \\
(4-14)\end{array}$ & $\begin{array}{c}21(29 / 139) \\
(14-29)\end{array}$ & $\begin{array}{c}21(29 / 138) \\
(15-29)\end{array}$ & $\begin{array}{l}7(11 / 147) \\
(4-13)\end{array}$ \\
\hline
\end{tabular}

CFQ, Chalder Fatigue Questionnaire; SF-36-PF, Short Form 36-item measure of physical function; CGI, Clinical Global Impression change measure; APT, adaptive pacing therapy; CBT, cognitive behaviour therapy; GET, graded exercise therapy; SMC, specialist medical care; CDC, Centers for Disease Control and Prevention; CFS, chronic fatigue syndrome; ME, myalgic encephalomyelitis; CI, confidence interval.

Normal range for CFQ was $\leqslant 18 / 33$; normal range for SF-36-PF was $\geqslant 60 / 100$.

Values given as $\%(n /$ total $)$.

status. For this reason, we made a post-hoc decision to model the more complete data set of those meeting the trial definition of recovery rather than the illness definition of recovery.

Table 2 shows the proportions who met the trial definition of recovery in subgroups that met alternative definitions of CFS or ME at baseline. The pattern of results was very similar to those for all participants; CBT and GET were associated with the largest proportions of participants recovered.

Table 3 shows that odds of trial definitions of recovery after either CBT or GET were more than three times those after either APT or SMC. There was no significant difference between APT and SMC. A similar pattern of differences was observed in the two subgroups that met the International (CDC) definition for CFS (interaction term $p$ value $=0.77$ ) and in those who met the London criteria for ME at entry (interaction term $p$ value $=0.76$ ).

\section{Discussion}

We found that CBT and GET were both significantly more likely than APT and SMC to be associated with recovery at 52 weeks, even when using a conservative definition of recovery. Between a fifth and a quarter 
Table 2. Composite criteria for trial recovery in subgroups meeting alternative definitions of CFS or ME at baseline

\begin{tabular}{lllll}
\hline & APT & CBT & GET & SMC \\
\hline $\begin{array}{l}\text { All participants } \\
\begin{array}{l}\text { International (CDC) } \\
\text { criteria }\end{array}\end{array}$ & $9(12 / 149)$ & $22(32 / 143)$ & $22(32 / 143)$ & $7(11 / 150)$ \\
$\begin{array}{l}\text { London ME criteria } \\
\text { (1) }\end{array}$ & $19(17 / 89)$ & $22(20 / 93)$ & $6(6 / 98)$ \\
\hline
\end{tabular}

CFS, Chronic fatigue syndrome; ME, myalgic encephalomyelitis ; APT, adaptive pacing therapy; CBT, cognitive behaviour therapy; GET, graded exercise therapy; SMC, specialist medical care; CDC, Centers for Disease Control and Prevention.

Values given as $\%(n /$ total $)$.

Table 3. Comparison of odds for composite trial recovery adjusted for baseline characteristics

\begin{tabular}{|c|c|c|c|c|c|c|}
\hline & \multicolumn{2}{|l|}{ All participants } & \multicolumn{2}{|c|}{$\begin{array}{l}\text { Met international (CDC) } \\
\text { criteria at baseline }\end{array}$} & \multicolumn{2}{|c|}{$\begin{array}{l}\text { Met London ME criteria at } \\
\text { baseline }\end{array}$} \\
\hline & OR $(95 \%$ CI $)$ & $p$ value & OR $(95 \% \mathrm{CI})$ & $p$ value & OR $(95 \%$ CI $)$ & $p$ value \\
\hline CBT $v . \mathrm{APT}$ & $3.36(1.64-6.88)$ & 0.001 & $2.73(1.16-6.44)$ & 0.022 & $2.72(1.09-6.78)$ & 0.032 \\
\hline CBT v. SMC & $3.69(1.77-7.69)$ & $<0.001$ & $4.14(1.56-11.00)$ & 0.004 & $3.18(1.23-8.23)$ & 0.017 \\
\hline GET $v$. APT & $3.38(1.65-6.93)$ & 0.001 & $2.96(1.27-6.90)$ & 0.012 & $2.52(1.01-6.28)$ & 0.048 \\
\hline GET v. SMC & $3.71(1.78-7.74)$ & $<0.001$ & $4.50(1.72-11.79)$ & 0.002 & $2.95(1.14-7.61)$ & 0.026 \\
\hline $\mathrm{APT} v . \mathrm{SMC}$ & $1.10(0.47-2.58)$ & 0.83 & $1.52(0.52-4.46)$ & 0.450 & $1.17(0.40-3.43)$ & 0.77 \\
\hline
\end{tabular}

CBT, Cognitive behaviour therapy; APT, adaptive pacing therapy; SMC, specialist medical care; GET, graded exercise therapy; CDC, Centers for Disease Control and Prevention; ME, myalgic encephalomyelitis; OR, odds ratio; CI, confidence interval.

International (CDC) interaction $p=0.77$; London ME interaction $p=0.76$.

of participants were recovered by 52 weeks after either CBT or GET, with an NNT of seven. A similar pattern was seen in the two subgroups meeting alternative definitions for CFS and ME at entry into the trial.

The main limitation of this analysis is the absence of a generally agreed measure of recovery. We addressed this by using multiple domains of health and disability. The domains chosen and the criteria for recovery on each were defined before we undertook the analysis. Alternative domains could have been used, such as return to work or objective measures of physical activity. Return to work is not, however, an appropriate measure of recovery if the participant was not working before their illness and is influenced by other factors such as the job market. Objective measures of physical activity have been found previously to correlate poorly with self-reported outcomes (Wiborg et al. 2010), which may be related to the finding that activity patterns in CFS patients are heterogeneous, with only a minority being pervasively passive (van der Werf et al. 2001). We did not include any measures of mood in our domains of recovery as mood is not part of the definition of the illness.

The amount of missing outcome data was greater after CBT and GET than after APT and SMC, but the percentages missing were small enough not to warrant sensitivity analyses, particularly because all but 33 (5\%) participants contributed some data. The prevalence of the case-level International (CDC) definition of CFS may have been inaccurate because we only examined for accompanying symptoms in the previous week, not the previous 6 months. The assessments of caseness (CDC, London and Oxford criteria) relied on a mixture of self-ratings and research assistant assessments, making some observer bias possible. We changed some of the thresholds for measuring recovery from those of the original protocols (White et al. 2007); we made the changes before analysis and to more accurately reflect recovery. Our finding that only $7 \%$ recovered after the minimal treatment of SMC, exactly the same proportion as the median recovery rate found without treatment (Cairns \& Hotopf, 2005), supports these revised thresholds. 
Finally, we cannot be sure that recovery was sustained beyond the assessment at 52 weeks.

How do these results compare with previous studies? We are not aware of any previous studies that have compared comprehensively defined recovery between different treatments. Two studies of recovery in adults after CBT found similar proportions in recovery: $23 \%$ and $24 \%$ (Deale et al. 2001; Knoop et al. 2007), compared with $22 \%$ in the PACE trial. One of these studies had a 5-year follow-up period rather than the 1 year of our study, and the majority had received further treatment in those extra 4 years, all patients being treated at one specialist CFS centre (Deale et al. 2001). The other study used similar criteria and domains for recovery (Knoop et al. 2007), but the definition for normal range used was the more liberal population mean \pm 2 s.D. rather than the more conservative 1 S.D. that we used; the treatment was delivered by therapists in one specialist CFS centre and outside of a trial setting. A meta-analysis of randomized controlled trials of CBT for CFS reported that a mean of $50 \%$ of the patients improved to the point of no longer being clinically fatigued (Malouff et al. 2008). A 2-year follow-up study after an educational intervention to encourage GET found that $55 \%$ of the treated patients no longer fulfilled trial criteria for CFS (Powell et al. 2004). Although not directly comparable, we found that $41 \%$ and $33 \%$ were within the population range for fatigue after CBT and GET respectively, although these proportions drop further when added to functional improvement; $54 \%$ and $56 \%$ of participants no longer met the trial entry (Oxford) case definition for CFS after CBT and GET. Our finding that $22-56 \%$ of participants met various composite or single criteria for recovery or improvement a year after starting either CBT or GET is therefore consistent with previously published studies. The NNT of 7 for recovery after both CBT and GET is within the range of the effects found for drug treatments in both general medical and psychiatric conditions (Leucht et al. 2012). Although only $22 \%$ recovered after either CBT or GET, if different participants recovered after CBT than after GET, then the proportion recovering after either treatment would be larger than $22 \%$, but not larger than $39 \%$. Recovery after CBT may be better in adolescents (Nijhof et al. 2012). The 7\% and 8\% recovered after both APT and SMC were similar to the $7 \%$ reported in a systematic review after no treatment, suggesting a lack of efficacy of these treatments (Cairns \& Hotopf, 2005).

The proportions recovered in each treatment arm were similar in the subgroups meeting alternative definitions of CFS and ME, implying that these findings generalize to different definitions of CFS and ME. Patients who have either CFS or ME characterized by a principal complaint of fatigue, and who are attending out-patient clinics, should therefore be offered either CBT or GET to provide the best chance of recovery with these treatments.

As a little more than a fifth of participants treated with CBT or GET had recovered a year after starting treatment, we still need to consider ways of enhancing the effectiveness of these treatments. Two ways of doing this could be to increase the number of sessions above that offered in the PACE trial (15 sessions), because a recent meta-analysis found that higher numbers of sessions improved efficacy (Castell et al. 2011), or enhancing delivery of therapy, such as over the internet (Nijhof et al. 2012). Another approach may be to offer both CBT and GET in series. A different approach would be to identify the factors that mediate the effect of these treatments, with the aim of optimizing their effectiveness; the mediation analysis of the PACE data is under way. CFS is a heterogeneous condition and we need to find ways of identifying subgroups that respond best to each type of therapy (Cella et al. 2011). Finally, we also need to develop additional forms of treatment.

In conclusion, recovery from CFS is more likely to occur when CBT or GET is added to SMC than after adding APT or giving SMC by itself. The relatively small proportion of recovered patients may reflect the heterogeneity of CFS; it should also spur us on both to enhance currently available therapies and to develop new and better treatments.

\section{Appendix. The PACE Trial Management Group}

The PACE Trial Management Group consisted of the authors of this paper plus (in alphabetical order): B. J. Angus, H. Baber, J. Bavinton, M. Burgess, L. V. Clark, D. L. Cox, J. C. DeCesare, E. Feldman, P. McCrone, G. Murphy, M. Murphy, H. O'Dowd, T. Peto, L. Potts, R. Walwyn, D. Wilks.

\section{Acknowledgements}

The funders of the PACE trial were: the UK Medical Research Council, the Department of Health for England, the Department for Work and Pensions, and the Scottish Chief Scientist Office. We thank Dr V. Schlezak for contributing to the literature review. Trial registration: ISRCTN54285094.

\section{Declaration of Interest}

P.D.W. has undertaken voluntary and paid consultancy work for the UK government and a reinsurance company. T.C. has received royalties from Sheldon Press and Constable \& Robinson. M.S. has 
undertaken voluntary and paid consultancy work for the UK government and consultancy work for an insurance company, and has received royalties from Oxford University Press.

\section{References}

Bowling A, Bond M, Jenkinson C, Lamping DL (1999). Short Form 36 (SF-36) Health Survey Questionnaire: which normative data should be used? Comparisons between the norms provided by the Omnibus Survey in Britain, the Health Survey for England and the Oxford Healthy Life Survey. Journal of Public Health Medicine 21, 255-270.

Cairns R, Hotopf M (2005). A systematic review describing the prognosis of chronic fatigue syndrome. Occupational Medicine 55, 20-31.

Castell BD, Kazantzis N, Moss-Morris RE (2011). Cognitive behavioural therapy and graded exercise for chronic fatigue syndrome: a meta-analysis. Clinical Psychology: Science and Practice 18, 311-324.

Cella M, Chalder T (2010). Measuring fatigue in clinical and community settings. Journal of Psychosomatic Research 69, 17-22.

Cella M, Chalder T, White PD (2011). Does the heterogeneity of chronic fatigue syndrome moderate the response to cognitive behaviour therapy? Psychotherapy and Psychosomatics 80, 353-358.

Chalder T, Berelowitz G, Hirsch S, Pawlikowska T, Wallace P, Wessely S (1993). Development of a fatigue scale. Journal of Psychosomatic Research 37, 147-153.

Deale A, Husain K, Chalder T, Wessely S (2001). Long-term outcome of cognitive behaviour therapy versus relaxation therapy for chronic fatigue syndrome: a 5-year follow-up study. American Journal of Psychiatry 158, 2038-2042.

Edmonds M, McGuire H, Price JR (2004). Exercise therapy for chronic fatigue syndrome. Cochrane Database of Systematic Reviews 3, CD003200.

Guy W (1976). ECDEU Assessment Manual for Psychopharmacology, pp. 218-222. National Institute of Mental Health: Rockville, MD.

Kamper SJ, Stanton TR, Williams CR, Maher CG, Hush JM (2011). How is recovery from low back pain measured? A systematic review of the literature. European Spine Journal 20, 9-18.

Knoop H, Bleijenberg G, Gielissen MF, van der Meer JWM, White PD (2007). Is a full remission possible after cognitive behavioural therapy for chronic fatigue syndrome? Psychotherapy and Psychosomatics 76, 171-176.

Leucht S, Hierl S, Kissling W, Dold M, Davis JM (2012). Putting the efficacy of psychiatric and general medicine medication into perspective: review of meta-analyses. British Journal of Psychiatry 200, 97-106.

Malouff JM, Thorsteinsson EB, Rooke SE, Bhullar N, Schutte NS (2008). Efficacy of cognitive behavioral therapy for chronic fatigue syndrome: a meta-analysis. Clinical Psychology Review 28, 736-745.
McAteer A, Elliott AM, Hannaford PC (2011). Ascertaining the size of the symptom iceberg in a UK-wide communitybased survey. British Journal of General Practice 61, e1-e11.

McHorney CA, Ware JE, Raczek AE (1993). The MOS 36-Item Short-Form Health Survey (SF-36): II. Psychometric and clinical tests of validity in measuring physical and mental health constructs. Medical Care 31, 247-263.

NICE (2007). Chronic Fatigue Syndrome/Myalgic Encephalomyelitis (or Encephalopathy). Diagnosis and Management of CFS/ME in Adults and Children. National Institute for Health and Clinical Excellence (NICE) Clinical Guideline 53 (www.nice.org.uk/nicemedia/pdf/ CG53QuickRefGuide.pdf).

Nijhof SL, Bleijenberg G, Uiterwaal CSPM, Kimpen JLL, van de Putte EM (2012). Effectiveness of internet-based cognitive behavioural treatment for adolescents with chronic fatigue syndrome (FITNET): a randomised controlled trial. Lancet 379, 1412-1418.

Nisenbaum R, Jones JF, Unger ER, Reyes M, Reeves WC (2003). A population-based study of the clinical course of chronic fatigue syndrome. Health and Quality of Life Outcomes 1, 49.

Powell P, Bentall RP, Nye FJ, Edwards RHT (2004). Patient education to encourage graded exercise in chronic fatigue syndrome 2-year follow-up of randomised controlled trial. British Journal of Psychiatry 184, 142-146.

Price JR, Mitchell E, Tidy E, Hunot V (2008). Cognitive behaviour therapy for chronic fatigue syndrome in adults. Cochrane Database of Systematic Reviews 3, CD001027.

Prins JB, van der Meer JW, Bleijenberg G (2006). Chronic fatigue syndrome. Lancet 367, 346-355.

Reeves WC, Lloyd A, Vernon SD, Klimas N, Jason LA, Bleijenberg G, Evengard B, White PD, Nisenbaum R, Unger ER; International Chronic Fatigue Syndrome Study Group (2003). Identification of ambiguities in the 1994 chronic fatigue syndrome research case definition and recommendations for resolution. BioMed Central Health Services Research 3, 25.

Sharpe MC, Archard LC, Banatvala JE, Borysiewicz LK, Clare AW, David A, Edwards RH, Hawton KE, Lambert HP, Lane RJ, Mann A, McDonald L, Mowbray J, Pearson D, Pelosi A, Peto T, Preedy V, Smith A, Smith D, Taylor D, Tyrrell D, Wallace P, Wessely S, White PD (1991). A report- chronic fatigue syndrome: guidelines for research. Journal of the Royal Society of Medicine 84, 118-121.

Tyrrell DAJ (1994). Report from the National Task Force on Chronic Fatigue Syndrome (CFS), Post Viral Fatigue Syndrome (PVFS) and Myalgic Encephalomyelitis (ME). Westcare: Bristol.

van der Werf SP, Prins JB, Vercoulen JHMM, van der Meer JWM, Bleijenberg G (2001). Identifying physical activity patterns in chronic fatigue syndrome using actigraphic assessment. Journal of Psychosomatic Research 49, 373-379.

White PD, Goldsmith KA, Johnson AL, Potts L, Walwyn R, DeCesare JC, Baber HL, Burgess M, Clark LV, Cox DL, Bavinton J, Angus BJ, Murphy G, Murphy M, O’Dowd H, 
Wilks D, McCrone P, Chalder T, Sharpe M; PACE trial management group (2011). Comparison of adaptive pacing therapy, cognitive behaviour therapy, graded exercise therapy, and specialist medical care for chronic fatigue syndrome (PACE) : a randomised trial. Lancet 377, 823-836.

White PD, Sharpe MC, Chalder T, DeCesare JC, Walwyn R; PACE trial group (2007). Protocol for the PACE trial: a randomised controlled trial of adaptive pacing, cognitive behaviour therapy, and graded exercise, as supplements to standardised specialist medical care versus standardised specialist medical care alone for patients with the chronic fatigue syndrome/myalgic encephalomyelitis or encephalopathy. BioMed Central Neurology 7, 6 .

Wiborg JF, Knoop H, Stulemeijer M, Prins JB, Bleijenberg G (2010). How does cognitive behaviour therapy reduce fatigue in patients with chronic fatigue syndrome? The role of physical activity. Psychological Medicine 40, 1281-1287. 